\title{
Visión de los MOOC desde una perspectiva práctica
}

\section{MOOC from a practical perspective}

\author{
Juan Carlos Aguado Franco \\ Universidad Rey Juan Carlos, URJC (España) \\ Coordinador del Monográfico
}

\section{Resumen}

A pesar de que la bibliografía existente acerca de los MOOC es muy abundante, todavía quedan sin resolver muchas incógnitas referentes a su utilidad actual y su evolución futura. Es necesario continuar realizando estudios que nos arrojen luz al respecto, especialmente si con ellos somos capaces de cuantificar e interpretar correctamente los datos recopilados. En este monográfico contamos con ocho aportaciones que nos ofrecerán una mejor visión de los MOOC desde una perspectiva práctica.

Palabras clave: nuevas tecnologías; enseñanza a distancia; abandono de estudios; enseñanza superior.

\begin{abstract}
Although the literature about MOOCs is abundant, yet many questions remain unresolved regarding their current usefulness and their future evolution. It is necessary to continue with studies that shed light about it. We must to be able to quantify and correctly interpret the collected data. In this issue we have eight papers that will give us a better view of the MOOCs from a practical perspective.
\end{abstract}

Keywords: ICT; distance learning; study dropout; higher education.

\section{INDICADORES DEL INTERÉS DE LOS MOOC}

En números anteriores de la Revista Iberoamericana de Educación a Distancia, ya ha sido abordado el estudio del fenómeno de los MOOC bajo diferentes enfoques. Así, se hizo con una visión centrada en "la filosofía educativa de los MOOC y la educación universitaria” presente en el volumen 18, número 2, o anteriormente en el volumen 17, número 1, con el título de "Entornos abiertos en la nueva educación a distancia”, amén de diferentes artículos publicados con temática similar en otros números, como por ejemplo, el artículo editorial del volumen 18, número 1. 
En efecto, algunos de los primeros artículos publicados en RIED con esta temática serían los de Soares, 2013; Torres y Gago, 2014; Marauri, 2014 y Tovar y Lesko, 2014, a los que han seguido muchos más. Si ampliamos el marco podemos observar que la bibliografía relacionada con los MOOC existente en revistas del ámbito de la educación o de la tecnología es extensísima, como se mostrará y analizará detenidamente en varios artículos de este monográfico.

A pesar de ello, todavía permanecen sin responder de manera tajante algunas de las numerosísimas preguntas que se planteaban en el trabajo de García-Aretio, 2015, relativas a si los MOOC son ¿eficaces?, ¿ंeficientes?, ¿un fraude?, ¿̇ostenibles?, ¿rentables?, ¿alternativa o complemento a la formación tradicional?, ¿una amenaza o una oportunidad para la Universidad tal como la conocemos hoy?, ¿realmente se aprende con los MOOC?, etc.

En este monográfico vamos a intentar dar respuesta a algunas de ellas. Para no centrarnos únicamente en un análisis meramente descriptivo, incorporaremos en la medida que sea posible estudios en los que pueda introducir alguna cuantificación. A este respecto, uno de los principales factores cuantitativos que se suelen considerar en relación con el impacto de los MOOC, tanto desde el aporte a la mejora del aprendizaje y la difusión del conocimiento, como en relación con los potenciales beneficios económicos que puedan generar, es el que hace referencia al número de inscritos y a la tasa de finalización de los cursos.

En efecto, uno de los aspectos más mencionados de los MOOC en la literatura, especialmente para cuestionar el interés que despiertan, se centra en las altas estadísticas de deserción, dado que solo un porcentaje mínimo de aquellos que inician un MOOC lo terminan (Belanger y Thorton, 2013; Koller et al., 2013; Yang, D. et al., 2013; Armstrong, 2014; Jordan, 2014; Poy y Gonzales-Aguilar, 2014; FidalgoBlanco et al., 2015).

En ese sentido, si queremos analizar esas tasas de abandono, estableciendo un paralelismo fácilmente comprensible, podemos observar que existen obras literarias que es preciso leer desde la primera hasta la última página para poder comprenderlas y valorarlas, partiendo del planteamiento inicial de la trama, siguiendo por el nudo y llegando hasta el desenlace. Otras, sin embargo, constan de capítulos independientes que se pueden disfrutar sin considerar los que los anteceden ni los que los preceden.

Así, por ejemplo, no es lo mismo una novela policíaca que un recetario de cocina. En efecto, si un lector únicamente ojea las primeras páginas de una novela, o si tras leer las primeras páginas decide saltar hasta el final para descubrir quién era el asesino, estará perdiéndose buena parte del disfrute de la lectura y la experiencia probablemente le resultará insatisfactoria, pues no estará siguiendo todo un desarrollo que es fundamental para la comprensión de la obra. Por el contrario, si se trata de un libro de cocina, una persona puede estar interesada en la receta de la paella valenciana pero no en la del cocido madrileño.

Si extrapolamos este símil a lo que ocurre con los MOOC, veremos que algunos se han de seguir desde el primer módulo hasta el último pues necesitan de una visión 
de conjunto para que el aprendizaje sea el adecuado y no es posible saltarse algunos módulos, pues unos y otros están enlazados, y la comprensión de los siguientes se vería perjudicada. En otros MOOC, sin embargo, un usuario puede ir al módulo que le interese para obtener la información que busca, y no pasar necesariamente por los demás módulos. Si es un curso de microeconomía, por ejemplo, un usuario con unos conocimientos mínimos puede ir directamente al módulo del monopolio, si es ese el tema que le interesa, y comprender lo que allí se explica sin la obligación de pasar por el módulo de la competencia perfecta ni por el del oligopolio. Del mismo modo, si necesita recordar cómo se utiliza un test no paramétrico como la $\mathrm{U}$ de Mann-Whitney o el test de Wilcoxon, no es necesario que comience en un MOOC de estadística por un primer módulo donde le expliquen qué es la media, la moda o la desviación típica.

Esos usuarios que van directamente al módulo que les interesa, por tanto, según las estadísticas, habrán comenzado ese MOOC pero no lo habrán terminado, y la tasa de finalización global del MOOC caerá. Esto último, por tanto, no nos estará informando obligatoriamente de la falta de éxito de ese MOOC, de que se trata de un curso que carece de interés. De hecho, el MOOC puede haber satisfecho perfectamente las necesidades formativas de esas personas y haberles proporcionado con exactitud y precisión los conocimientos que buscaban.

Para analizar desde un punto de vista práctico la importancia y el papel que desempeñan en el presente los MOOC y el rol que pueden llevar a cabo en el futuro dentro del sistema educativo, no es suficiente con valorar ni el número de inscritos ni las tasas de finalización de los estudiantes, sino que sería necesario contar con indicadores que nos hablen del nivel de satisfacción del usuario más allá de los datos descritos anteriormente.

En algunos artículos de este monográfico se intentará aplicar este enfoque, buscando medir cuantitativamente determinadas variables, pero también la correcta interpretación de los datos obtenidos relativos a distintos aspectos de los MOOC.

\section{SOBRE EL ACTUAL NÚMERO DE RIED}

El presente monográfico, que lleva por título "Visión de los MOOC desde una perspectiva práctica", viene a ahondar en el estudio de este tipo de cursos, pretendiendo ofrecer un conocimiento más exhaustivo y más cercano de la realidad del mundo de los MOOC, intentando en la medida de lo posible obtener datos cuantitativos con los que poder medir y analizar el impacto de estos cursos en la educación.

Los dos primeros artículos de este monográfico están orientados hacia el análisis de la producción científica relativa a los MOOC. Así, los profesores Santiago Mengual-Andrés, Esteban Vázquez-Cano y Eloy López Meneses, de las Universidades de Valencia, UNED y Pablo de Olavide respectivamente, en su artículo titulado "La productividad científica sobre MOOC: aproximación bibliométrica 2012-2016 
a través de SCOPUS", analizan la productividad científica del fenómeno MOOC a partir del análisis de 752 publicaciones indexadas en la base de datos SCOPUS en el periodo 2012-2016. En este artículo podremos apreciar cuál es la evolución que ha experimentado en los últimos años, cuáles son los autores y trabajos que reciben un mayor número de citas, los principales países que generan literatura al respecto, etc., ofreciéndonos en definitiva una visión panorámica muy precisa acerca de los principales aspectos relacionados con los estudios publicados acerca de los MOOC.

En una línea concomitante con la del trabajo anterior, en su artículo titulado "Análisis de la literatura científica sobre los cursos en línea abiertos y masivos (MOOC)", Airton Zancanaro y María José Carvalho de Souza Domingues, de la Universidade Regional de Blumenau FURB (Brasil), se plantean mapear las investigaciones acerca de MOOC publicadas en dos bases de datos científicas: Scopus y Web of Science, dado que identifican la necesidad de que se realicen estudios que apunten los realizados previamente y sugieran pautas para futuras investigaciones. Además, dada la enorme cantidad de trabajos que se publican con esta temática, resulta muy interesante la labor efectuada por estos autores orientada a realizar una clasificación en macrotemas de los trabajos identificados o la identificación de las referencias más utilizadas por los autores. Ambas tareas facilitarán la realización de estudios posteriores, que los autores de este artículo consideran necesarios al constatar que es un tema que tiene todavía un prometedor recorrido por delante.

Dado el carácter iberoamericano de la revista, hemos considerado muy oportuno incluir a continuación el artículo titulado "La producción académica en portugués sobre recursos educativos en abierto", de los profesores Airton Zancanaro, de la Universidade Regional de Blumenau, y Tel Amiel, de UNICAMP, instituciones ambas de Brasil. El objetivo de su trabajo consiste en detectar y analizar la literatura académica sobre los recursos educativos en abierto publicada en portugués, con la ayuda de bases de datos y revistas científicas que utilizan técnicas bibliométricas.

Conocida por tanto la productividad existente en la literatura acerca de los MOOC con los tres artículos anteriores, parece conveniente plantearse a continuación cuál es el nivel de satisfacción -entre otras variables- que han experimentado los participantes en los MOOC. Para ello, contamos con el trabajo de Ángel González de la Fuente, de Telefónica Educación Digital, y de David Carabantes Alarcón, profesor de la Universidad Complutense de Madrid, titulado "MOOC: medición de satisfacción, fidelización, éxito y certificación de la educación digital".

Estos autores han analizado los datos proporcionados por dos encuestas realizadas a usuarios de distintos MOOC alojados en la plataforma MiríadaX. El trabajo no se limita a cuantificar la satisfacción de las expectativas a través de una encuesta, sino que aúna también el análisis de la fidelización de los alumnos inscritos al expresar cuál es su grado de interés en seguir cursando MOOC. En sus conclusiones, valoran los MOOC como un formato de éxito para el presente y el futuro de la adquisición de conocimiento en línea. 
Es en este sentido, donde cobra más importancia el artículo que se presenta a continuación, titulado "¿Pueden los MOOC favorecer el aprendizaje, disminuyendo las tasas de abandono universitario?” del profesor Juan Carlos Aguado Franco, de la Universidad Rey Juan Carlos de Madrid, España. En efecto, en este trabajo podemos ver, con datos muy concretos, de qué manera el uso de un MOOC como un instrumento de apoyo al aprendizaje y para la adquisición de conocimientos, puede resultar muy útil para alumnos oficiales matriculados en la Universidad, tanto en la modalidad presencial como para aquellos que recurren a la formación a distancia. El trabajo se justifica en el hecho de que el abandono de los estudios provoca unos costes tanto para quien decide desertar como para la sociedad en su conjunto, dado que se han invertido una serie de recursos que no han conducido a cristalizar en una titulación. El artículo nos muestra un altísimo nivel de satisfacción y de aceptación de la metodología utilizada por parte de los alumnos involucrados en la experiencia, además de unos resultados académicos notablemente mejorados que ayudarán a la disminución de la tasa de abandono de los estudios.

Ese coste económico que se produce con el abandono de los estudios entronca directamente con el siguiente artículo, en el que la profesora Nuria González Rabanal, de la Universidad de León, España, nos muestra un análisis de los factores determinantes de la oferta y la demanda de los MOOC, en su artículo titulado "Cursos MOOC: un enfoque económico". Destaca en su trabajo que los MOOC han sido concebidos como bienes libres, accesibles a cualquier persona en cualquier lugar y de forma gratuita -de ahí la "O" de open- lo que no impide que ya se haya suscitado un debate relacionado con su éxito y su potencial beneficio económico. Finaliza el trabajo analizando cómo el beneficio económico directo o indirecto de los MOOC está fundamentado en una nueva forma de negocio, en la que las fuentes de ingresos están diversificadas.

Posteriormente, la profesora Beatriz Sedano Cuevas, de la UNED, nos muestra en su artículo titulado "La atención a las necesidades y demandas específicas del alumnado en un mundo globalizado: el caso de un MOOC de español para viajar", que aunque es un ámbito de creciente expansión, los MOOC relacionados con la enseñanza de lenguas extranjeras son pocos y con pocas investigaciones científicas realizadas al respecto. En su caso, el MOOC se encuadra dentro del proyecto europeo ECO, y está dirigido hacia estudiantes de español que desean aprender una lengua para viajar. El trabajo realizado parte de un necesario análisis previo de las necesidades y las expectativas de los alumnos participantes, lo que, a través de las encuestas realizadas al inicio y final del curso, se revela como un factor determinante para el éxito de este tipo de iniciativas.

Por último, se abordan los aspectos prácticos y técnicos acerca de todo el proceso encaminado a la fabricación de un MOOC. Así, en el artículo titulado "Metodología de producción para el desarrollo de contenidos audiovisuales y multimedia para MOOC", realizado por los profesores Manuel Gértrudix, Mario Rajas y Sergio Álvarez, todos ellos de la Universidad Rey Juan Carlos de Madrid, explican cómo es necesario 
para crear un MOOC de calidad llevar a cabo un proceso de planificación, diseño y desarrollo en el que se establezcan metodologías específicas que aúnen estrategias y técnicas procedentes de distintos ámbitos, como el diseño de recursos educativos, la producción audiovisual y el desarrollo multimedia e interactivo.

De esta forma, los profesores que coordinan un MOOC no tienen que ocuparse de todos los aspectos técnicos de elaboración de los contenidos audiovisuales y de cualquier otro tipo, al poder contar con un equipo que se ocupe de esos aspectos. Nos aportan los autores de este artículo, en este sentido, su valiosa experiencia práctica en la puesta en marcha de los cinco primeros MOOC alojados en la plataforma URJCx, para la que ha sido necesaria la creación de equipos de trabajo especializados y que se ha llevado a cabo con un proceso que ha desembocado en la generación de un modelo de producción más ágil.

\section{REFLEXIÓN FINAL}

Mucho se ha escrito ya acerca de los MOOC, y mucho más se escribirá en los años venideros sobre ellos, pues todavía permanecen muchas preguntas sin responder acerca de la utilidad y del papel que los MOOC desempeñarán en el futuro. La evolución que experimenten en los próximos años, de hecho, nos dirá hacia dónde se dirigen. De momento, no parece que haya universidades que hayan visto comprometida su supervivencia debido a que sus potenciales alumnos prefieran cursar MOOC de otras prestigiosas instituciones. En efecto, en el mercado laboral, hoy por hoy, se valora más un título universitario que los MOOC que se hayan podido cursar impartidos por una universidad de renombre.

Por el contrario, como veremos en algún artículo de este monográfico, existen casos en los que los MOOC se utilizan como un valioso complemento de la formación oficial reglada impartida en centros oficiales, suponiendo una oportunidad más que una amenaza para las universidades.

Dada la variada temática que abordan los MOOC, por otro lado, tampoco se puede afirmar en muchos casos que su objetivo sea facilitar la inserción laboral de quienes los cursan, sino que ayudan a ampliar el abanico de conocimientos que se pueden obtener con este tipo de cursos, sin estar orientados necesariamente hacia la capacitación para el empleo.

Si nos fijamos en el perfil de los alumnos, además, podemos apreciar que un porcentaje muy elevado lo componen personas de mediana edad, que ya cuentan con una titulación universitaria, y que ocupan un puesto de trabajo. Se trata de personas, por tanto, que desean refrescar conocimientos o que tienen curiosidad por temas que no necesariamente han de estar ligados directamente con el desarrollo de su carrera profesional.

Los MOOC por tanto han venido para quedarse, pero únicamente con el transcurrir del tiempo podremos observar cuál es el papel que desempeñan finalmente 
en el sistema educativo, pues todavía quedan sin resolver muchos interrogantes al respecto.

El presente número de RIED se completa con otros artículos miscelánea que, aunque no forman parte del monográfico "Visión de los MOOC desde una perspectiva práctica", suponen aportaciones interesantes al campo de estudio propio de esta revista centrada en propuestas educativas de carácter no presencial.

\section{REFERENCIAS BIBLIOGRÁFICAS}

Armstrong, L. (2014). 2013-the Year of Ups and Downs for the MOOCs. Changing Higher Education. Recuperado de http:/ goo.gl/SqwGWn

Belanger, Y., y Thornton, J. (2013). Biolectricity: A Quantitative Approach. Duke's University's First MOOC. Recuperado de http:/ dukespace.lib.duke.edu/dspace bitstream/handle/10161/6216/Duke Bioelectricity MOO C Fall2012.pdf

Fidalgo-Blanco, A. et al. (2015) Methodological Approach and Technological Framework to Break the Current Limitations of MOOC Model. Journal of Universal Computer Science, 21, 712-734 doi: 10.3217/jucs-021-050712

García Aretio, L. (2015). MOOC, ¿tsunami, revolución o moda pasajera? RIED. Revista Iberoamericana de Educación a Distancia, 18(1), 9-21. Recuperado de http://revistas.uned.es/index.php/ried/ article/view/13812/12492

Jordan, K. (2014). Initial trends in enrolment and completion of massive open online courses. The International Review of Research in Open and Distance Learning, 15, 1. Recuperado de http://www.irrodl. org/index.php/irrodl/article/view/1651

Koller, D., Ng, A., Do, C., y Chen, Z. (2013). Retention and Intention in Massive Open Online Courses: In Depth. Educause Review. Recuperado de http://goo.gl DEJzxZ
Marauri, P. M. (2014). Figura de los facilitadores en los cursos online masivos $\mathrm{y}$ abiertos (COMA/MOOC): nuevo rol profesional para los entornos educativos en abierto. RIED. Revista Iberoamericana de Educación a Distancia, 17(1), 35-67.

Poy, R., y Gonzales-Aguilar, A. (2014). Factores de éxito de los MOOC: algunas consideraciones críticas. RISTI. Revista Ibérica de Sistemas de Tecnología de la Información, 1(3), 95-118. Recuperado de http://www.academypublisher.com risti/no13/risti13.pdf

Soares, M. J. (2013). Proposições e controvérsias no conectivismo. RIED. Revista Iberoamericana de Educación a Distancia, 16(2), 9-31.

Torres, D., y Gago, D. (2014). Los MOOCs y su papel en la creación de comunidades de aprendizaje y participación. RIED. Revista Iberoamericana de Educación a Distancia, 17(1), 13-34.

Tovar, E., y Lesko, I. (2014). Analysis of successful modes for the implementation and use of Open Course Ware (OCW) \& Open Educational Resources (OER) in higher education. The virtual mobility case. RIED. Revista Iberoamericana de Educación a Distancia, 17(1), 131-148.

Yang, D., Sinha, T., Adamson, D., y Rose, C. P. (2013). Turn On, Tune in, Drop Out: Anticipating Student Dropouts in Massive Open Online Courses. Recuperado de https://goo.gl/r32q95 


\section{PERFIL ACADÉMICO Y PROFESIONAL DEL COORDINADOR}

Juan Carlos Aguado Franco. Licenciado en Ciencias Económicas por la UAM, Master of Science por la Universidad de Montpellier, Francia, y Doctor en Economía por la URJC. Profesor de Fundamentos del Análisis Económico desde hace más de 20 años, es uno de los primeros creadores de MOOC en España e Iberoamérica, con su MOOC de microeconomía del que ha realizado ya 7 ediciones desde su puesta en marcha en enero de 2013. Es director también de los MOOC, de macroeconomía y de teoría de juegos en MiríadaX, y director del taller "La magia de la Economía". Autor de seis libros, su investigación se centra, por un lado, en el estudio microeconómico del comportamiento de los agentes económicos, y por otro, en el análisis de la forma de introducir eficazmente nuevas metodologías docentes en los procesos formativos. E-mail: juancarlos.aguado@urjc.es

\section{DIRECCIÓN DEL AUTOR}

Facultad de Ciencias Jurídicas y Sociales (URJC)

Paseo de los Artilleros s/n, 28032

Madrid (España)

Fecha de recepción del artículo: 26/10/2016

Fecha de aceptación del artículo: 27/10/2016

\section{Como citar este artículo:}

Aguado Franco, J. C. (2017). Visión de los MOOC desde una perspectiva práctica. RIED. Revista Iberoamericana de Educación a Distancia, 20(1), pp. 31-38. doi: http://dx.doi.org/10.5944/ried.20.1.17481 